\title{
Asymmetric Dense Breast is an Independent Breast Cancer Risk Factor
}

\begin{abstract}
Alikhassi $\mathbf{A}^{\mathbf{1}}$, Shariatalavi $\mathbf{R}^{\mathbf{1}}$ and Moradi $\mathrm{B}^{\mathbf{2}}$ ${ }^{1}$ Department of Radiology, Cancer Institute, Imam Khomeini Hospital, Tehran University of Medical Sciences, Tehran, Iran

${ }^{2}$ Department of Radiology, Advanced Diagnostic and Interventional Radiology Research Center, Imam Khomeini Hospital Complex, Tehran University of Medical Sciences, Tehran, Iran

*Corresponding author: Afsaneh Alikhassi, Department of Radiology, Cancer Institute, Imam Khomeini Hospital, Tehran University of Medical Sciences, Tehran, 3314114197, Iran
\end{abstract}

Received: April 20, 2021; Accepted: May 11, 2021; Published: May 18, 2021

\begin{abstract}
Objectives: There are multiple known breast cancer risk factors, but most women with breast cancer do not have any of them, so there should be some other unknown risk factors. We hypothesized that asymmetric breast densities could be another breast cancer risk factor.
\end{abstract}

Method: In this study, we defined two case and control groups with 136 women with breast cancer and 136 who did not have breast cancer, respectively. Any different type of asymmetry in either breast was recorded in both groups.

Result: The frequency of focal asymmetry in cases was $47(34.6 \%)$, which was statistically more significant than in the control group (28 $(20.6 \%)$ ) $(p=0.010)$. There were three $(2.9 \%)$ and five $(3.7 \%)$ global asymmetries in the case and control groups, respectively $(p=0.735)$. The frequency of one view asymmetry in the case and control groups was not significant $(16(11.8 \%)$ and $9(6.6 \%)$ respectively) $(p=0.142)$. In the case group, $59(43.4 \%)$ women had at least one type of asymmetry, compared to $41(30.1 \%)$ in the control group $(p=0.02)$. We identify focal asymmetries (likelihood ratio, $1.215 ; p=0.027)$ is risk factors for breast cancer.

Conclusion: Breast density asymmetry is a breast cancer risk factor that could be scored, thus enhancing risk stratification for screening and prevention.

Keywords: Predictive factors of cancer; Asymmetric dense breast; Mammographic breast density

\section{Introduction}

Breast cancer is the most common cancer among women, and mammography is the main modality of breast cancer screening [1]. Mammographic Breast Density (MBD), which reflects the amount of fibroglandular tissue, is an independent risk factor of breast cancer, and women with MBD of $75 \%$ or more have four to six times greater chance of developing breast cancer than women with MBD of $\leq 10 \%$ $[2,3]$. MBD can mask cancer on mammography and lower sensitivity [4].

In the most recent version of the ACR BIRADS reporting system, the percentage density is no longer used, and the type of breast composition is stressed. The sensitivity of mammography for noncalcified lesions decreases as the BI-RADS breast density category increases [4].

We hypothesis that the phenotype of mammography could be a risk factor for breast cancer. By this, we mean that breasts with more asymmetric fibroglandular tissue have more chance of future cancer. Not only the amount of breast tissue, but also the pattern of breast tissue are breast cancer risk factors. Asymmetry represents unilateral deposits of fibroglandular tissue not conforming to the definition of a mass.

The goal of our study was to determine whether asymmetric breast density is associated with breast cancer.

\section{Methods}

We conducted a case-control study among women referred to
Imam Khomeini Hospital in Tehran University of Medical Science, which consist of known cases of breast cancer and healthy non-cancer patients who were referred for 2D digital screening mammography. This study was approved by the ethical committee of the Tehran University of Medical Science with the reference number of IR.TUMS. IKHC.REC.1397.281.

One-hundred-thirty-six women with breast cancer who were referred to our hospital were randomly selected as the case group and 136 who did not have breast cancer were considered as the control group and matched for age, breast density and menopausal status. In this study, the breast cancer group was those patients who had a confirmed pathology of breast cancer after tissue sampling. The median time from index mammogram in the case group to diagnosis for breast cancer was 1 to 2 years.

Exclusion criteria were previous history of surgery, biopsy, or hormone therapy. Written informed consent was taken from all the included patients to use their mammography information without declaring their personal data. Mammographies of all patients were reviewed by two radiologists with breast subspecialty who were blind to the assignment of patients to the case and control groups.

Any type of asymmetry in either breast, according to the last version of ACR BIRADS [4], was documented in case and control groups by both radiologists separately and in case of any disagreement, it was discussed and recorded: asymmetry was considered an area of fibroglandular tissue visible on only one mammographic projection; global asymmetries were those asymmetries consisting of an
Austin J Womens Health - Volume 8 Issue 2 - 2021

Submit your Manuscript | www.austinpublishing group.com

Alikhassi et al. (C) All rights are reserved
Citation: Alikhassi A, Shariatalavi R and Moradi B. Asymmetric Dense Breast is an Independent Breast Cancer Risk Factor. Austin J Womens Health. 2021; 8(2): 1051. 



Figure 1: Screening mammography, mediolateral view, right (a) and left (b); no asymmetry is seen in the either side.
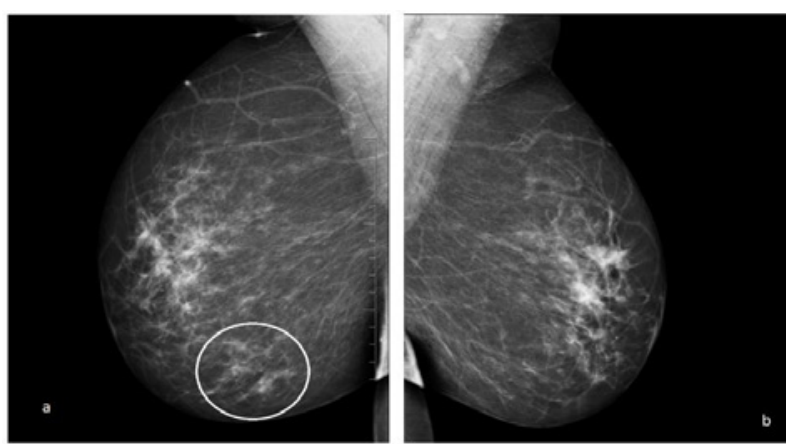

Figure 2: Screening mammography, mediolateral projection of right (a) and left (b) breast; an asymmetry is seen in lower part of right breast.

asymmetry over at least one-quarter of the breast; focal asymmetries referred to non-symmetrical density that was visible in both of the mammogram standard views and that did not have the characteristics of the mammary mass; and developing asymmetries those that were new, larger, and more conspicuous than on a previous examination (Figure 1 and 2).

Of note, in routine mammography reports, stable asymmetries are not reported, while in this study, based on our goal, all kinds of asymmetries, whether stable or not, were documented.

The results for quantitative variables were expressed as mean and standard deviation (mean \pm SD) and for the qualitative variables in percentages. Comparisons of quantitative variables were performed by independent t-test. Comparisons between qualitative variables were done using the Chi-square test. A multivariate logistic regression model was used to determine the predictive factors for malignancy in the presence of underlying factors, such as focal asymmetry in mammography. Finally, the diagnostic value of the asymmetry presence in mammography in the prediction of breast cancer was analyzed. Data were analyzed by SPSS version 20 and SAS version 9.1. A significance level less than 0.05 was considered.

\section{Results}

We included 136 patients with known cancer and 136 healthy patients referred for screening mammography. In the control group, the average age was $45.48 \pm 10.87$ years, compared to $50.07 \pm 7.07$ in the case group. In both groups, ten patients (7.4\%) had a positive family history $(\mathrm{p}>0.05)$.
The case group had more focal asymmetries than the control group (47 (34.6\%) vs. 28 (20.6\%), p=0.010). The average age in patients with and without focal asymmetries were 46.07 with 9.48 $\mathrm{SD}$ and 48.43 with $9.36 \mathrm{SD}$ respectively, and it was not significant (p value $=0.065$ ).

There were three $(2.9 \%)$ global asymmetries among the case group and five $(3.7 \%)$ among the control group $(\mathrm{p}=0.735)$. In one view asymmetry, frequency in the case and control group were not significantly different: $16(11.8 \%)$ and $9(6.6 \%)$ respectively, with $\mathrm{p}$ value (0.142).

In the case group, 59 (43.4\%) women had at least one type of asymmetry, compared to $41(30.1 \%)$ in the control group ( $\mathrm{p}=0.02)$. Table 1 shows the frequencies of different types of asymmetries in both groups. Patients with cancer had more asymmetries than healthy women $(\mathrm{p}=0.030)$.

We used multivariate logistic regression model analysis with multiple variables to predict factors associated with breast cancer. We found that focal asymmetries (likelihood ratio, 1.215; $\mathrm{p}=0.027$ is possible risk factors for breast cancer.

\section{Discussion}

In the available literature, a variety of clinical and imaging predictors of breast cancer has been proposed. Age, specific gene mutations (e.g., BRCA) and positive family history are among known risk factors [5]. However, most patients with breast cancer do not have known risk factors [6].

We know that screening has some disadvantages, including falsepositive cases and negative biopsies [7]. To increase the efficacy of breast cancer screening, it will need to become personalized. The age for starting screening, the intervals, and the need for additional modalities are factors that could be defined based on the patient's risk factor profile. So, having a powerful and applicable risk assessment model is valuable [8].

Density in mammography is among the known risk factors for breast cancer. Women with high mammographic density have four- and six-times higher lifetime risk of developing breast cancer than age-matched women with low breast density $[9,10]$. Of note, mammographic density is also associated with genetic risk factors and family history of breast cancer. Increased density in mammography also decreases the sensitivity of screening $[11,12]$.

Table 1: Frequency of various types of asymmetries in case and control groups

\begin{tabular}{|c|c|c|c|}
\hline Characteristics & $\begin{array}{c}\mathbf{P} \\
\text { Value }\end{array}$ & $\begin{array}{c}\text { Control groups } \\
(\mathbf{N}: \mathbf{1 3 6})\end{array}$ & $\begin{array}{c}\text { Case groups (N: } \\
\mathbf{1 3 6})\end{array}$ \\
\hline Focal asymmetry & 0.01 & $28(20.6 \%)$ & $47(34.6 \%)$ \\
\hline Focal asymmetry Number & 0.03 & & \\
\hline $\mathbf{0}$ & & 108 cases $(79.4 \%)$ & 89 cases $(65.4 \%)$ \\
\hline $\mathbf{1}$ & & 23 cases $(16.9 \%)$ & 39 cases $(28.7 \%)$ \\
\hline $\mathbf{2}$ & & 4 cases $(2.9 \%)$ & 8 cases $(5.9 \%)$ \\
\hline Other & & 1 case $(0.7 \%)$ & 0 cases $(0 \%)$ \\
\hline $\begin{array}{c}\text { Global asymmetry } \\
\text { Asymmetry }\end{array}$ & 0.735 & 5 cases $(3.7 \%)$ & 3 cases $(2.9 \%)$ \\
\hline $\begin{array}{c}\text { Presence of at least one type } \\
\text { of asymmetry }\end{array}$ & 0.02 & 41 cases $(30.1 \%)$ & 59 cases $(43.4 \%)$ \\
\hline
\end{tabular}


The phenotype of the fibroglandular tissues in breasts might be a biomarker of future breast cancer. We believe that in addition to density, asymmetric fibroglandular tissue is an additional risk factor for breast cancer. In humans, there is bilateral symmetry in paired morphological traits, including breasts [13], and we as radiologists evaluate the bilateral mammographic tissue symmetry and any possible change over time as a tool to early diagnose breast cancer. This study shows that a patient with cancer, overall, had more asymmetries than a healthy woman $(\mathrm{p}=0.02)$ and having focal asymmetries (likelihood ratio, 1.215; $\mathrm{p}=0.027$ ) is also a possible risk factor for breast cancer.

In the study by Bin Zheng et al., computed bilateral mammographic density asymmetry was the strongest risk factor (AUC=0.719 \pm 0.027 ) with a significantly higher prediction power than woman's age $(p=0.022)$ and mammographic density assessed by either radiologists (ACR BIRADS density) $(\mathrm{p}<0.001)[14]$.

The previous available studies worked on the computed quantitative global bilateral asymmetry of mammographic density. In this study, we detected a high diagnostic value of qualitative breast asymmetries, based on the ACR definition in predicting breast cancer. Unlike focal asymmetry that was associated with breast cancer, the global asymmetry did not have such a diagnostic value. However, the frequency of global asymmetry was low in both groups, and thus we would need more cases to properly assess the diagnostic value of global asymmetry.

Based on our findings, we propose that a scoring system that includes breast density asymmetry can be an effective, and noninvasive tool, which can help find and follow-up at-risk patients.

\section{Conclusion}

Given the availability and cost-effectiveness of screening mammography, tracking those people most at risk of breast cancer according to mammographic findings is of great importance. The presence of asymmetry in breast composition is highly diagnostic for the prediction of breast cancer. Therefore, it is hoped that more studies will be done with larger sample sizes to investigate asymmetry and other mammary risk factors associated with breast cancer.

\section{References}

1. Bray F, Ren JS, Masuyer E, et al. Estimates of global cancer prevalence for 27 sites in the adult population in 2008. Int J Cancer. 2013; 132: 1133-1145.

2. Boyd NF, Guo H, Martin LJ, et al. Mammographic density and the risk and detection of breast cancer. N Engl J Med. 2007; 356: 227-236.

3. Engmann NJ, Golmakani MK, Miglioretti DL, et al. Population-attributable risk proportion of clinical risk factors for breast Cancer. JAMA Oncol. 2017; 3: 1228-1236

4. Sickles, EA, D’Orsi CJ, Bassett LW, et al. ACR BI-RADS ${ }^{\circledR}$ Mammography. In: ACR BI-RADS ${ }^{\circledR}$ Atlas, Breast Imaging Reporting and Data System. Reston, VA, American College of Radiology. 2013.

5. Amir E, Freedman OC, Seruga B, et al. Assessing women at high risk of breast cancer: a review of risk assessment models. J Natl Cancer Inst. 2010; 102: 680-691.

6. Madigan MP, Ziegler RG, Benichou J, et al. Proportion of breast cancer cases in the United States explained by well-established risk factors. JNCI. 1995; 87: 1681-1685

7. Seely JM, Alhassan T. Screening for breast cancer in 2018-what should we be doing today? Current oncology (Toronto, Ont.). 2018; 25: S115-S124.

8. Schousboe JT, Kerlikowske K, Loh A, et al. Personalizing mammography by breast density and other risk factors for breast cancer: Analysis of health benefits and cost-effectiveness. Ann Intern Med. 2011; 155: 10-21.

9. Phoebe E. Freer. Mammographic Breast Density: Impact on Breast Cancer Risk and Implications for Screening. RadioGraphics. 2015; 35: 302-315.

10. Boyd NF, Martin IJ, Stone J, et al. Mammographic densities as a marker of human breast cancer risk and their use in chemoprevention. Curr Oncol Rep. 2001; 3: 314-321.

11. Vachon CM, Sellers TA, Carlson EE, et al. Strong evidence of a genetic determinant for mammographic density, a major risk factor for breast cancer Cancer Res. 2007; 67: 8412-8418.

12. Martin LJ, Melnichouk $\mathrm{O}$, Guo $\mathrm{H}$, et al. Family history, mammographic density, and risk of breast cancer. Cancer Epidemiol Biomarkers Prev. 2010; 19: 456-463.

13. Scutt D, Lancaster GA, Manning JT. Breast asymmetry and predisposition to breast cancer. Breast Cancer Research. 2006; 8: R14.

14. Zheng B, Sumkin JH, Zuley ML, et al. Bilateral mammographic density asymmetry and breast cancer risk: a preliminary assessment. European journal of radiology. 2012; 81: 3222-3228. 